\title{
Complex Composite Odontome
}

\author{
Neha Talwar ${ }^{1}$, Abhimanyu Airon², Devanshu Rohilla ${ }^{3}$, Divya Nirjhawan³ ${ }^{3}$ R. K. Airon ${ }^{5}$
}

${ }^{1}$ Department of Medicine, MMIMSR (MM Deemed to be University), Mullana, Haryana, India. ${ }^{2}$ Department of Medicine, MMIMSR (MM Deemed to be University), Mullana, Haryana, India. ${ }^{3}$ Department of Radiology, MMIMSR (MM Deemed

to be University), Mullana, Haryana, India. ${ }^{4}$ Department of Radiology, MMIMSR (MM Deemed to be University), Mullana, Haryana, India. ${ }^{5}$ Department of Radiology, MMIMSR (MM Deemed to be University), Mullana, Haryana, India.

\section{INTRODUCTION}

An odontome results due to the abnormal development of tooth forming tissues. The term complex composite odontome is used for a tumour in which calcified dental tissues are irregularly arranged, bearing no morphological similarity to a tooth. Compound composite odontome consists of a group of small denticles or malformed teeth. Odontome can be discovered at any age in any part of the mandibular or maxillary arches. They often prevent the eruption of teeth and, as they are small, they may be discovered only during routine examination. Another odontome described is Dens - in-dente (Dilated odontome, Gestant odontome, Dens Invaginatus). During the course of development, a part of enamel organ may become invaginated and on completion of development the affected tooth contains an enamel lined cavity. The permanent lateral maxillary incisor is often involved in the mild form of invagination. Caries and pulp infection can develop before the tooth complete eruption. The presence of the enamel lined cavity within a tooth gives a complicated appearance on a section resembling a tooth within a tooth (Hence term dens in-dente) Fourth type of Odontome described is Ameloblastic Odontome. It consists of odontogenic epithelium, enamel and Dentine. It is more commonly seen in children and sometimes in adults. It is more frequent in maxilla and seen as a well-defined translucent area with irregular opacities in it. It is difficult to differentiate it from complex composite odontome.[1]

\section{PRESENTATION OF CASE}

A 20-year-old male came to the surgery OPD of a hospital with swelling on the left lower jaw for 2 years. Swelling was painless and has diffuse border involving angle of mandible and was firm in consistency. Overlying skin was normal. All vital signs were found to be normal. Intra oral examination revealed diffuse swelling in the left mandibular region. Lips, tongue, floor of the mouth and palate were normal. Patient was advised $\mathrm{x}$-ray mandible PA and left lateral views. X-ray mandible showed radiopaque mass with surrounding radiolucent area. Radiopaque mass was uniform in density which was more than the bone density. Diagnosis of complex composite odontome was made on the basis of radiological findings. Biopsy confirmed the radiological diagnosis. Patient refused surgery.
Corresponding Author:

R. K. Airon,

Department of Radiology,

MMIMSR (MM Deemed to be University), Mullana-133207, Dist- Ambala,

Haryana, India

E-mail: rkairon1947@gmail.com

DOI: $10.14260 / j e m d s / 2019 / 709$

Financial or Other Competing Interests: None.

How to Cite This Article:

Talwar N, Airon A, Rohilla D, et al. Complex composite odontome. J. Evolution Med. Dent. Sci. 2019;8(43):3273-3275, DOI: 10.14260/jemds/2019/709

Submission 29-08-2019,

Peer Review 08-10-2019,

Acceptance 15-10-2019,

Published 28-10-2019. 


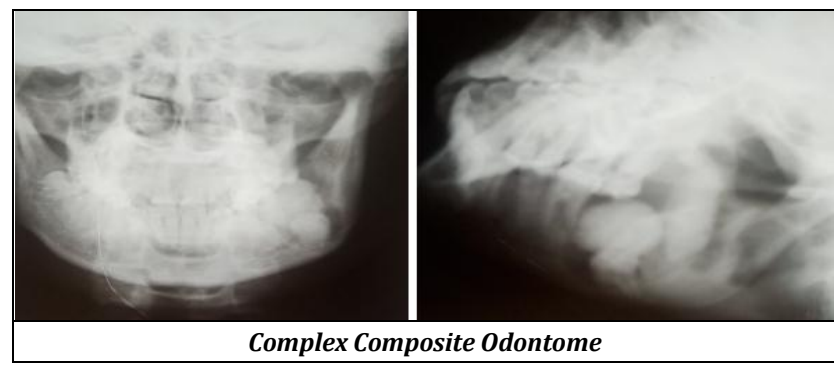

\section{DISCUSSION}

Odontomes are considered to be developmental anomalies resulting due to the growth of differentiated epithelial and mesenchymal cells. The odontomes can be best defined as a hamartoma than a true neoplasm. The second edition of the WHO histological typing of odontogenic tumour, divides odontomes under the broad category of tumour containing odontogenic ectomesenchyme and odontogenic epithelium with or without dental tissue formation.

\section{This Classification Consist of Three Types of Odontomes}

1. Odontoameloblastoma

2. Complex odontome

3. Compound odontomes. ${ }^{[2]}$

Odontomes are common odontogenic tumours containing all the structures of tooth forming tissues. Odontomes constitute about $22 \%$ of all odontogenic tumours of the jaws. They are common benign odontogenic tumours of mesenchymal and epithelial origin. The majority of odontomes in the anterior segment of the jaws are compound composite type (61\%) and majority of odontomes in the posterior segment of jaw are complex composite type (34\%). Both types of odontomes are found more commonly on the right side of the jaw than on the left side. Compound composite odontome are more commonly seen in incisor cuspid region of the upper jaw whereas complex composite odontome are found in molar and premolar region of the mandible. Odontomes are commonly seen in the region of permanent dentition.

The exact aetiology odontome is not known. It may occur due to trauma which may get infected. Odontome may be found in any tooth bearing region of the jaw. Majority of complex composite odontome are seen in the mandible. The authors also published most common classification given by world health organization (WHO).

\section{WHO Classification}

"One of the most common classification is given by World Health Organisation. Four lesions containing enamel and Dentine of normal appearance are defined in the WHO classification. They are as follows-

1. Ameloblastic fibro-odontome: Consist of varying amounts of calcified dental tissue and dental papilla-like tissue, the latter component resembling fibroma. The ameloblastic fibro-odontome is considered as an immature precursor of complex odontome.

2. Odonto-ameloblastoma: It's a very rare neoplasm which resembles an ameloblastoma both structurally and clinically but contains enamel and dentine.
3. Complex odontome: when the calcified dental tissues are simply arranged in an irregular mass bearing no morphologic similarity to rudimentary teeth

4. Compound odontome: Composed of all odontogenic tissues in an orderly pattern that result in many teeth like structures but without morphologic resemblance to normal teeth."[3]

Junqueral et al reported a rare case of intraosseous odontoma erupted into the oral cavity arising from the posterior maxilla in a 23-year-old male. On histopathological examination this case came out to be the complex odontoma.[4]

Butnick Steven D. reviewed 149 cases of odontomas and accepted 65 cases from literature. He observed that odontomas were most commonly diagnosed in $2^{\text {nd }}$ decade of life. $65 \%$ of all odontomas occurred in maxilla. Compound odontomas were seen most commonly in anterior maxilla and complex odontomas were frequently seen in posterior jaw. Males were more commonly involved. Common presenting symptoms were an impacted tooth with retention of deciduous teeth. ${ }^{[5]}$

Regezi et al studied 706 odontogenic tumours from a total of 54534 oral biopsy specimens. They noted that odontomas consisted of more than $65 \%$ of odontogenic tumours, out of which $10 \%$ were ameloblastomas and remaining 6 categories of odontogenic tumours accounted for $25 \%$ of the lesions. A possible variant of calcifying epithelial odontogenic tumour was reported.[6]

Katz R W published a study of 396 odontomas and cases were divided into 1 of three categories using commonly accepted histological criteria for compound complex form. They noted that compound odontomas were most common type $(70 \%$ of all cases). They were commonly seen in the age group of 11-15 years. Maxillary incisor area or canine area of either jaw was commonly involved. Sex distribution was equal. Complex odontomas were seen in similar age group and sex distribution. They occurred commonly in molar region. Complex odontomas were associated with unerupted teeth more than compound odontomas. The study observed correlation between the site of odontoma and the age of treatment.[7]

Bruno Al Meida et al published a case of dilated odontome in the posterior mandible. Patient was 47 years old female having history of episodes of diffuse pain and discomfort in the left posterior mandibular region. Intraoral x-ray showed an intraosseous circular formation with radio opaque external limits and radiolucent interior. Computed tomography scan showed the bone integrity around the formation and independence of the neuro vascular structures of the region. Possibility of the dilated odontome was given which was proved on histopathological examination. ${ }^{[8]}$

\section{CONCLUSIONS}

A good clinical examination and required radiographs usually provide a good diagnosis. Missed diagnosis will complicate the treatment plan. Hence it is the moral duty of clinician and radiologist to evaluate the lesion thoroughly and make a correct diagnosis. 


\section{REFERENCES}

[1] Maria GME. The teeth and jaws. In: Lewis HK, eds. A text book of X-ray diagnosis. Vol. I. $4^{\text {th }}$ edn. London: 1969: p. 461-547.

[2] Siddhartha SV, Freny K. Complex odontome: a review \& case report. International Journal of Advanced Health Sciences 2014;1:21-5.

[3] Satish V, Prabhadevi MC, Sharma R. Odontome: a brief overview. International Journal of Clinical Pediatric Dentistry 2011;4(3):177-85.

[4] Junquera L, de Vicente JC, Roig P, et al. Intraosseus odontoma erupted into the oral cavity: an unusual pathology. Med Oral Pathol Oral Cir Bucal 2005;10(3):248-51.
[5] Budnick SD. Compound and complex odontomas. Oral Surgery Oral Medicine Oral Pathology 1976;42(4):501-6.

[6] Regezi JA, Kerr DA, Courtney RM. Odontogenic tumors: analysis of 706 cases. Journal of Oral Surgery 1978;36(10):771-8.

[7] Katz RW. An analysis of compound and complex odontomas. ASDC journal of Dentistry for Children 1989;56(6):445-9.

[8] Almeida B, Silva A, Pereira M, et al. Case report of a dilated odontome in the posterior mandible. International Journal of Surgery Case Reports 2016;20:14-6. 